\title{
Appropriation des cultures académiques dans la mobilité internationale : analyse des difficultés rencontrées par des doctorants internationaux de l'UFC de Besançon
}

Résumé. Alors que la mobilité internationale au niveau doctoral fournit plus de $40 \%$ des étudiants inscrits dans les laboratoires français (INSEE, 2017), cette population a peu suscité l'intérêt des chercheurs, tant en anthropologie qu'en didactique (Bouchard, 2010; Fløttum \& Vold, 2010; Reuter, 1998, 2004). La rédaction d'une thèse respectant les attendus de l'institution d'accueil représente pourtant une entreprise considérable pour ces chercheurs, audelà des seules difficultés linguistiques. Cet article, issu de notre propre travail doctoral, se penche sur les implications de la mobilité académique au niveau de la recherche. Une enquête de type ethnographique tente de mettre au jour les difficultés rencontrées dans la rédaction d'une thèse en sciences du langage par un groupe de doctorants internationaux, ainsi que les stratégies mobilisées pour les surmonter. Le résultat vise à mieux cerner la situation de ces jeunes chercheurs afin de faciliter l'insertion des futurs doctorants internationaux dans leur communauté scientifique d'accueil.

Mots-clés : mobilité doctorale, cultures académiques, transfert, stratégies, didactique de l'écrit universitaire.

Abstract. Even though international mobility provides more than $40 \%$ of PhD students in France (INSEE, 2017), few specialists of language teaching or anthropology paid attention to this population (Bouchard, 2010; Fløttum \& Vold, 2010; Reuter, 1998, 2004). Nevertheless, the writing of a PhD dissertation according to the expected standards of the host university is a significant undertaking to these junior researchers, far and beyond linguistic difficulties. In this article, written from our own thesis, we consider the consequences of academic mobility at doctoral level. An ethnographic-based investigation tries to disclose the problems that a doctoral students group has to deal with during the writing of their dissertation in linguistics, as well as the strategies they use to overcome it. The results aim to a better understanding of their situation, which could ease future international $\mathrm{PhD}$ students' insertion in their host scientific community.

Keywords: doctoral mobility, academic cultures, transfer, strategies, learning and teaching academic writing.

Notice biographique. *Enseignant au « Centre de linguistique appliquée » (Besançon, France), doctorant en didactique des langues et des cultures / plurilinguisme et didactique des langues étrangères (DILTEC-EA 2288), à l'Université Sorbonne Paris Cité sous la direction de Valérie Spaëth, et au Dépt de plurilinguisme et de didactique des langues étrangères, Université de Fribourg, Suisse, sous la direction d'Aline Gohard-Radenkovic, dans le cadre d'une co-tutelle entre les deux universités. 
Short Biography. *Teacher at "Centre de linguistique appliquée" (Besançon, France), $\mathrm{PhD}$ student in the Research Group of Foreign Languages \& Cultures Learning and Teaching and of Multilingualism (DILTEC-EA 2288) at the Sorbonne Paris Cité University, under the supervision of Prof. Dr Valérie Spaëth and at the Dept of Multilingualism and of Foreign Languages Learning and Teaching, Fribourg University, Switzerland, under the supervision of Prof. Dr Aline Gohard-Radenkovic in the frame of "Cotutelle" between the two Universities. 


\section{Introduction}

Nous nous intéressons ici aux conditions d'études, et plus spécifiquement aux obstacles rencontrés par un groupe d'étudiants internationaux au cours de la rédaction de leur thèse de doctorat en sciences du langage, entreprise à l'université de Franche-Comté (Besançon, France), au sein de l'équipe d'accueil ELLIADD ${ }^{1}$. Ces doctorants, à nos yeux sujets scientifiques en mobilité, sont, pour la plupart, parvenus en France dans un contexte de mobilité académique non encadrée, poussés dans cette démarche par les aléas politiques et économiques vécus par leur pays d'origine. Bien que porteurs d'importants capitaux culturels et universitaires (Bourdieu, 1979), d'expérience (Gohard-Radenkovic \& Murphy-Lejeune, 2008), plurilingues et pluriculturels (Zarate, 2005), ils se heurtent, dès le début de leurs études doctorales, à une situation «extra-ordinaire » (Bouchard, 2010) sur laquelle peu de chercheurs spécialisés dans la mobilité se sont penchés. Comprendre leurs difficultés et tenter d'y remédier représente, il est vrai, de réels défis : comment donner des réponses à portée générale à un public marqué par des différences multiples? Ils sont, en effet, fortement spécialisés dans des domaines scientifiques divers, souvent abscons et difficiles d'accès; quoique s'inscrivant à un même niveau universitaire, leurs compétences langagières en français sont très hétérogènes ${ }^{2}$; issus de systèmes éducatifs et académiques souvent très éloignés de l'université française, leur appropriation des codes et usages de leur institution d'accueil, formant une «culture en soi » (Gohard-Radenkovic, 2002), est freinée par la difficulté des co-acteurs de la mobilité (GohardRadenkovic \& Rachédi, 2009), que sont les personnels enseignants et BIATOSS ${ }^{3}$, à les expliciter, à « (...) donner quelques clés pour la compréhension des pratiques sous-jacentes (ou 'habitudes de faire' inconsciemment intégrées et reproduites par les natifs) (...), qui aideront l'apprenant à s'approprier l'environnement du 'savoir-faire' (...)» (Gohard-Radenkovic, $1995: 8)$.

Notre recherche ${ }^{4}$, située au croisement des champs de la didactique du français langue étrangère et seconde (FLES) et de la didactique de l'écrit universitaire, vise à repérer et catégoriser les difficultés rencontrées par des doctorants internationaux dans la rédaction de leur thèse, ainsi qu'à proposer des moyens de les surmonter. Quels transferts implique le fait de réaliser une recherche dans un nouveau système universitaire et dans une langue étrangère ? Comment favoriser l'appropriation d'un habitus doctoral fait non seulement d'un discours, mais de postures scientifiques? On tentera ici, par l'analyse d'entretiens compréhensifs (Kaufmann) conduits avec les doctorants, de brouillons de thèses collectés auprès d'eux et d'entretiens

\footnotetext{
${ }^{1}$ EA 4661, Edition, Littératures, Langages, Informatique, Arts, Didactique, Discours. http://elliadd.univ-fcomte.fr/ ${ }^{2}$ Rappelons qu'en France, contrairement aux niveaux inférieurs, aucun prérequis en français n'est imposé au moment de l'inscription en doctorat. Le directeur de thèse et l'école doctorale prennent seuls la décision d'admettre un étudiant, le plus souvent sur son profil scientifique. Il n'est ainsi pas rare de voir un doctorant entreprendre une thèse avec un niveau B1 à l'écrit.

${ }^{3}$ Personnels de bibliothèques, ingénieurs, administratifs, techniques, ouvriers de service, sociaux et de santé, intervenant dans l'enseignement supérieur français.

${ }^{4}$ Cheminement épistémologique et discursif dans le processus d'écriture de recherche : Le cas des doctorants internationaux en sciences du langage à l'université de Franche-Comté. Thèse réalisée sous la direction de Valérie Spaëth (DILTEC-Paris III Sorbonne nouvelle) et d'Aline Gohard-Radenkovic (Université de Fribourg, Suisse) dans le cadre d'une co-tutelle.
} 
menés avec certains de leurs directeurs de recherches, de mieux comprendre les difficultés et les tensions dans lesquelles les place la situation de mobilité, ainsi que les stratégies déployées pour y remédier. Après avoir présenté les principaux concepts qui nous éclairent, les doctorants participant à cette étude et le contexte dans lequel ils travaillent, on tâchera de typifier les principales difficultés auxquelles ils se heurtent, ce qui permettra de tracer quelques pistes de remédiation linguistique, didactique et culturelle.

\section{Concepts et méthode d'analyse}

Nous travaillons dans une perspective socio-anthropologique et selon une approche qualitative. L'écrit universitaire, dans cette optique, est davantage considéré dans sa dimension sociale que textuelle. Trois éclairages contribuent à donner sens à nos corpus. La sociologie de l'éducation a montré les processus d'affiliation (Coulon, 1997) à l'œuvre dans l'insertion universitaire, tandis que les pratiques langagières académiques, la circulation internationale des concepts et les mécanismes de sélection ont été mis au jour par les travaux de Bourdieu \& Passeron (1970; Bourdieu, 1990). Par ailleurs, la réflexion épistémologique et philosophique s'est penchée sur les conséquences de la mobilité scientifique en redéfinissant les rôles du transfert et de la traduction (Cassin, 2004; Espagne, 2013), tout en réaffirmant le caractère situé de toute activité scientifique, rompant avec la croyance en l'universalité de la science (Latour \& Woolgar, 1988; Licoppe, 1996; Lévy-Leblond, 2006). L'héritage bachelardien et phénoménologique soustendant les pratiques scientifiques des sciences humaines en France est ainsi souligné. C'est enfin sur les travaux en didactique des langues et des cultures que nous nous appuyons pour caractériser l'écriture doctorale et penser les conditions de son apprentissage. Écrire, c'est pour nous connaître un ensemble de pratiques développées progressivement au sein d'une littératie (Goody, 1978) selon un continuum allant de l'école élémentaire à l'enseignement supérieur, et qui peuvent être transmises (Barré-de Miniac, Brissaud, \& Rispail, 2005) y compris au niveau universitaire (Pollet, 2012, 2014; Reuter, 2012). C'est l'appropriation de matrices rhétoriques (Gohard-Radenkovic, 1995) et disciplinaires (Weisser, 2010), souvent peu didactisées car incorporées et enfouies, qui conditionne la pratique d'une écriture de haut niveau. L'étude de la mobilité académique conduite depuis une vingtaine d'années enrichit notre regard sur les statuts de ses acteurs, perçus comme voyageurs (Murphy-Lejeune, 2003), aventuriers (Papatsiba, 2003), ou en cours de sédentarisation (Keller-Gerber, 2015). Leur présence a un effet révélateur (au sens photographique) de la «culture en soi »(Gohard-Radenkovic, 2002), des « dimensions cachées » (Hall, 1979), et du « savoir enculturé » (Gohard-Radenkovic, 1995, 1999, 2002; Cuko, 2016) circulant dans les institutions d'accueil.

L'analyse des corpus cherche à mettre au jour les caractéristiques de la littératie doctorale dans le contexte décrit, en conduisant une analyse de contenu (Bardin, 1977) des textes de guidage réunis. On dégage ainsi des normes linguistiques, communicatives, stylistiques (Allouche \& Maurer, 2010) attendues. Les conceptions de ce qu'est une thèse réussie en sciences du langage seront repérées par l'analyse d'entretiens compréhensifs (Kaufmann, 2007) semi-directifs, conduits avec quelques directeurs de recherche des doctorants participant à notre étude. 


\section{Des étudiants venus du monde entier à Besançon}

Nous avons choisi de construire plusieurs corpus autour de six doctorants qui ont accepté de participer à ce travail. Malgré la variété de leurs profils (langue maternelle, domaine de spécialité, niveau de maitrise du français, âge), plusieurs points communs les réunissent. Tous, non francophones natifs, sont ou se sont inscrits dans un processus de mobilité académique longue, diplômante, et non encadrée, afin de suivre des études doctorales en sciences du langage proposées par l'université de Franche-Comté à Besançon, au sein de l'école doctorale LECLA et de l'équipe d'accueil ELLIADD. Le tableau suivant (Figure 1) présente la situation de chacun d'entre eux.

\begin{tabular}{|c|c|c|c|c|c|c|}
\hline Pseudonyme & RAHMA & ABDER & SHAFIKA & BAHA & PACO & LI \\
\hline Pays & IRAK & EGYPTE & IRAK & LYBIE & PÉROU & CHINE \\
\hline Spécialité & $\begin{array}{l}\text { Didactique. } \\
\text { du FLE } \\
\text { /traductologie }\end{array}$ & $\begin{array}{l}\text { Analyse } \\
\text { de } \\
\text { discours }\end{array}$ & $\begin{array}{l}\text { Linguistique. } \\
\text { contrastive }\end{array}$ & $\begin{array}{l}\text { Linguistique } \\
\text { arabe }\end{array}$ & TAL & $\mathrm{TAL}$ \\
\hline $\begin{array}{l}\text { Langue } \\
\text { maternelle }\end{array}$ & $\begin{array}{l}\text { Arabe moyen- } \\
\text { oriental }^{5}\end{array}$ & $\begin{array}{l}\text { Arabe } \\
\text { égyptien }\end{array}$ & $\begin{array}{l}\text { Arabe } \\
\text { moyen- } \\
\text { oriental }\end{array}$ & $\begin{array}{l}\text { Arabe } \\
\text { maghrébin }\end{array}$ & Quechua & Mandarin \\
\hline $\begin{array}{l}\text { Directeur de } \\
\text { thèse } \\
\text { (pseudonyme) }\end{array}$ & M. Daniel & $\begin{array}{l}\text { Mme } \\
\text { Charles } \\
\text { / M. } \\
\text { Bernard }\end{array}$ & M. Daniel & M. Daniel & $\begin{array}{l}\text { M. } \\
\text { Arnaud }\end{array}$ & $\begin{array}{l}\text { M. } \\
\text { Arnaud }\end{array}$ \\
\hline
\end{tabular}

Quelles contingences ont amené ces doctorants à étudier à Besançon plutôt que dans une ville universitaire plus importante? Rappelons que les sciences du langage y sont étudiées depuis l'immédiat après-guerre, sous l'impulsion d'abord du lexicologue B. Quemada, fondateur du Centre de linguistique appliquée (1958), puis du linguiste J. Peytard, qui développe dès les années 1960 une riche tradition de recherche en analyse du discours, linguistique, sémiotique et didactique (Madini, 2010). La conjonction de ces deux facteurs (présence d'un centre universitaire d'enseignement du FLE et d'un laboratoire de recherche pionnier en sciences du langage) est à l'origine d'un flux d'étudiants internationaux, particulièrement au niveau doctoral, que la modicité du coût de la vie dans la capitale franc-comtoise et la proximité de Paris ont contribué à entretenir.

\footnotetext{
${ }^{5}$ Nous nous appuyons sur les découpages linguistiques effectués par l'INALCO (www.inalco.fr)
} 
Trois corpus seront exploités dans cet article : on a rassemblé un ensemble de textes rédigés par les étudiants, destinés à figurer dans le texte final de la thèse, mais encore relus par eux seuls (Corpus 1, brouillons de thèse). Leur analyse vise à repérer et catégoriser tous types de difficultés rédactionnelles et à connaître leurs difficultés effectives. Cependant, ce corpus serait à nos yeux peu compréhensible sans la connaissance du parcours de vie des auteurs, de leur motivation à entreprendre une thèse, de leur parcours éducatif et des logiques qui ont présidé à la rédaction, informations recueillies par entretiens dans le corpus 2 (entretiens avec les doctorants). Enfin, on a tenté de connaître les postures scientifiques et les normes rédactionnelles attendues des étudiants dans leur contexte par des entretiens avec leur directeur de thèse (corpus 3 entretiens avec les directeurs de recherche). On fera émerger des types de difficultés par le croisement et le dialogue entre ces trois corpus.

\section{Des obstacles et des stratégies}

La connaissance globale des sujets observés, permise par l'approche qualitative s'appuyant sur une perspective socio-anthropologique, aide à dépasser le niveau, superficiel à nos yeux, représenté par l'étude des phénomènes textuels et linguistiques. On verra dans ce qui suit à quel point, pour chaque type d'obstacle repéré, les parcours de vie, les processus rédactionnels et les postures scientifiques acquis dans deux contextes académiques différents s'entre-éclairent, démontrant la nécessité de ce type d'approche dans les études de didactique en contexte de mobilité.

\subsection{Trouver un directeur de thèse}

Pour Abder (corpus 2), il a été relativement facile de convaincre un enseignant-chercheur de l'encadrer, même s'il a dû se prêter à certaines vérifications ${ }^{6}$ :

ABDER : (...) j' ai fait ça / j'ai fait un [projet de ] thèse sur l'analyse sémiotique qui a (...) plu [à Mme Charles] / elle a dit c'est un bon travail / parce que moi j'ai envoyé mon mémoire [de Master] elle m'a dit ok / je / j'peux vous envoyer [la lettre d'] acceptation / tous les professeurs [que j'ai contactés] m'ont demandé un résumé pour mon mémoire de Master mais Mme [Charles] (...) a préféré de voir mon Master/ en entier (...) /elle m'a dit ok/ et aussi/ avant de de (...) parce que ce qui m'a (...) étonné / elle m'a / demandé de faire un/ un échange avec moi/ ORAL/ pour voir moi j'ai pensé ça c'est pour examiner / mon niveau de français/ elle m'a dit [Abder] on peut se (...) fixer un rendez-vous sur Skype (...) et après ce/ c't entretien-là/ Skype/ elle a dit ok (...)

Deux phases se dessinent de ce témoignage: une première prise de contacts large, dans plusieurs universités (tous les professeurs), suivie d'un examen en trois temps de la candidature par la directrice intéressée : projet scientifique (elle a dit c'est un bon travail), lecture des travaux précédents ([elle] a préféré de voir mon Master en entier), évaluation des capacités

\footnotetext{
${ }^{6}$ Conventions de transcription : / pause brève, // pause longue, CAPITALES : accent d'intensité, [entre crochets] : modification ou ajout (pseudonyme, précision, description d'un geste ou d'une attitude), (...) : coupure dans la transcription. Tours de parole : SF (Sébastien Favrat) ou pseudonyme (cf. Figure 1).
} 
langagières et scientifiques effectives par un entretien. Cependant, tous n'ont pas eu un parcours aussi simple. Etre admis à préparer une thèse semblait hors de sa portée à Li :

LI : chez nous étudiant en doctorat c'était un RÊVE/ j'ai jamais pensé que j'aurais étudié en doctorat/ mais avoir fait le Master tout ça / enfin si j'ai la chance de continuer je continue / j'ai/j'ai parlé avec plusieurs profs donc / finalement (...) on s'est mis d'accord sur un sujet pour travailler voilà SF : donc t'as pas eu de difficulté pour trouver un professeur qui te suive / en doctorat/ ça a pas été spécialement compliqué

LI : si si au début j'ai j'ai quand même cherché partout et puis j'ai j'ai eu plusieurs refus (...) j'ai cherché le domaine / enfin/ le projet que je porte (..) après j'ai cherché sur internet les profs mais/

SF : t'as cherché seulement à Besançon (...)

LI : oh/ aussi à l'extérieur de Besançon aussi un petit peu tout ça/ mais/ mais j'ai pas/ [trouvé]

L'émotion transparaît dans la relation de ce souvenir, de la représentation du doctorat comme niveau inaccessible (rêve, chance) et du désarroi vécu dans cette phase d'échecs (j'ai j'ai quand même cherché partout et puis j'ai j'ai eu plusieurs refus), jusqu'à la phase de sélection évoquée succinctement et avec pudeur (on s'est mis d'accord sur un sujet pour travailler voilà). Pourtant, c'est le parcours de Shafika qui illustre le mieux les écarts entre les postures scientifiques du candidat et les attendus du directeur de thèse au moment de l'inscription. L'étudiante, stagiaire du CLA, avait bénéficié de l'entremise d'un enseignant pour repérer un possible directeur de thèse, mais les premiers contacts semblent un dialogue de sourds :

SHAFIKA : (...) moi je viens avec cette idée (...) j'ai raconté tout ça à M. [Daniel] / il dit mais ça c'est/ c'est nul/ c'est normal/vous n'avez rien ajouté/ le doctorat c'est que vous inventez quelque chose/ et toi tu n'as rien inventé (...) il m'a dit [ton sévère et solennel] allez/ retournez dans votre chambre / et voici les références des écrivains qui ont écrit sur [ce sujet ${ }^{7}$ / tu dois lire (...) et après ça vous allez rédiger votre projet (...) et après ça / venez/ (...) moi j'ai dit/ après ça / je n'ai pas d'autre choix/ il faut que je convainque ce monsieur/ (...) il n'y a pas beaucoup de temps/

Une période de travail intense s'ouvre alors, doublée d'une pression administrative (les délais d'inscription approchent), et d'un décalage entre le statut de candidate au doctorat en France et celui d'enseignante en Irak :

SHAFIKA : les références qu'il m'a données (...) l'écriture est TRÈS TRÈS DURE / j'ai rien compris/ (...) j'ai envoyé un mail / (...) il me répond après quelques minutes (...) COMMENT vous êtes un doctorant et vous dites j'ai rien compris/ et comment vous avez tout lu / (...) ou bien vous n'avez rien lu / ou bien vous n'avez rien compris/ (...) moi cette lettre/ il me fait nerveuse / j'ai fait un message / (...) j'ai dit vous ne me connaissez pas qui suis-je / je vais vous raconter / peutêtre vous allez comprendre avec qui vous parlez/ moi j'ai raconté / tout ce que j'avais fait/ et finalement il m'a dit / j'attends toujours que vous terminiez votre lecture et après ça vous allez passer me voir (...) et j'attends toujours votre projet (...)

\footnotetext{
${ }^{7}$ Le sujet de la thèse de Shafika est effacé pour garantir son anonymat.
} 
Le projet de mobilité doctorale place Shafika dans une situation intenable: en tant que professeure de français elle doit maitriser cette langue, mais pour être admise en doctorat elle doit se soumettre à une épreuve en français dont elle ne comprend pas les règles, faute de quoi elle devra rentrer en Irak et admettre son échec. Elle ne peut trouver le dénouement à cette tension qu'en elle-même :

SHAFIKA : je m'enferme dans ma chambre/ (...) je ne réponds pas au téléphone à ma famille / parce que la famille//elle déchire ma tête (...) moi j'ai décidé/ d'oublier tout / concentrer sur ça / (...) j'ai passé deux mois en lisant / en cherchant / finalement/j' ai trouvé le sujet (...)/ je vais collecter mon corpus à Besançon à Paris à Lyon/ (...) et j'ai rédigé trois pages/ avec des références / j'ai envoyé un mail à M. [Daniel] (...) et j'ai réservé un billet d'avion pour rentrer en Irak/ (...) je vais là-bas [au rendez-vous] / je lui ai présenté le projet/ il a commencé à lire à lire à lire/ et il me regarde comme ça [regard fixe] il dit/ félicitations madame/ je vais écrire l'attestation MAINTENANT (...) moi j'ai pris le papier [claque des mains] je suis rentrée à mon pays/ j'ai commencé ma recherche

Dès les premiers pas, le parcours est donc tout sauf linéaire. Les difficultés ne s'arrêtent cependant pas une fois le sésame de l'inscription obtenu. Le rôle de chercheur reste encore à construire.

\subsection{Exposer sa recherche}

Connaître les normes organisationnelles de l'écrit universitaire est indispensable à la rédaction de la thèse, mais ces normes, détachées des matrices rhétoriques et des postures scientifiques qui leur donnent sens, suffisent-elles à donner forme à la thèse ? L'extrait suivant, situé à la fin de la revue de littérature de Paco (Corpus 1), fournit des éléments de réponse :

\section{CONCLUSION}

Concernant les dictionnaires français-quechua et quechua-français il existe très peu de documents sur papier.

L'orthographe reste encore incohérente entre les diverses sources publiées, même après l'officialisation de l'alphabet.

Le dictionnaire d'Itier respecte rigoureusement l'orthographe officielle et peut servir comme document de référence.

Le lexique répertorié dans les divers dictionnaires consultés est relativement réduit. Par exemple, le nombre de verbes simples ne dépasse pas 1100.

Aucun de ces documents n'est directement utilisable en tant que dictionnaire électronique car, outre le problème d'orthographe, ni les catégories grammaticales ni les propriétés morphologiques ne sont renseignées.

Cette conclusion intermédiaire frappe par son aspect éclaté et son manque de cohérence textuelle. L'absence de connecteurs logiques, de rituels démarcatifs (Allouche \& Maurer, 2010) 
éloigne stylistiquement ce texte des conclusions observées dans nos autres corpus ${ }^{8}$. Loin du paragraphe sémantique théorisé par Adam (2018), on observe ici un délitement du paragraphe qui oblige le lecteur à repérer lui-même les divergences et les points communs entre les ouvrages cités, ce qui ralentit sa lecture. Pendant l'entretien, on souhaite donc comprendre l'intention qui a amené Paco à rédiger ainsi :

SF : (...) est-ce qu'il y a une raison précise pour laquelle tu as fait une conclusion pour ton premier chapitre/ est-ce que tu en as senti la nécessité

PACO : non pas vraiment (...) c'est/ le FORMALISME dont on parlait tout à l'heure / il faut faire une conclusion / par chapitre (...) / parce que le premier chapitre ça montre une/ espèce d'introduction sur lequel / c'est pas du tout du TRAVAIL/ 'fin le travail il est pas là/ le travail c'est à partir du chapitre deux trois quatre

L'étudiant met en avant les contraintes extérieures qu'il a ressenties (un chapitre perçu comme inutile, l'obligation d'une conclusion...). Il ne voit pas le sens des normes qu'on lui impose, qui sont pour lui pur formalisme. La nécessité de guider le lecteur, manifestant le passage d'une écriture autocentrée à une écriture hétérocentrée, et nous paraît correspondre à ce que Flottum \& Vold (2010), nomment rôle de scripteur, lui semble étrangère. Ce cas précis montre comment une intervention didactique (en l'occurrence, un cours de méthodologie suivi par Paco, rappelant l'organisation générale de la thèse et le rôle des introductions et conclusions) peut se révéler contre-productive si elle se focalise sur le « comment » (rédiger, présenter, organiser...) sans expliciter le « pourquoi » des normes présentées.

Les brouillons analysés révèlent l'importance de l'obstacle représenté par l'appropriation inachevée de normes "plaquées » sur le texte, révélant la difficulté, pour les enseignants et formateurs, à expliciter les matrices rhétoriques qui sous-tendent l'écriture doctorale. Nous constatons cependant que celle-ci semble également régie, à un niveau plus profond, par un ensemble de postures de recherche enracinées dans ce qu'on appellera ici l'espace épistémologique constitué par l'université d'accueil.

\subsection{Se positionner en tant que chercheur}

Dans son cadre contextuel, Li (Corpus 1) rédige cette présentation sociolinguistique de son pays :

Lorsque nous parlons de la minorité, nous savons qu'en Chine est un pays immense dont la population, selon le groupe d'origine, peut avoir 56 ethnies différentes. Parmi ces 56 ethnies, l'ethnie Han représente $92 \%$ de la population, c'est pour cela nous appelons le chinois mandarin, qui est officiellement la langue de nation. Pour les autres ethnies, étant donné qu'ils couvrent

\footnotetext{
${ }^{8}$ Afin de cerner les caractéristiques d'une thèse réussie en sciences du langage, quatre thèses « modèles », indiquées par les directeurs de recherche, ont été rassemblées et analysées.
} 
seulement une petite partie de la population, nous les appelons" 'minorité", évidement pour certains minorités, ils ont leur propre langages pour s'écrire. [notre soulignement]

L'emploi du pronom énonciatif «nous » dans ce passage est intéressant. Certes, le «nous parlons de minorités » peut tout à fait correspondre au nous «scientifique » (défini comme «nous 1 » par Fløttum et Vold, op.cit : 44, c'est-à-dire « renvoyant plus directement au locuteur [que on]), quoiqu'il puisse également englober le lecteur. La situation est moins claire pour le second «nous» : est-ce bien le chercheur qui nomme cette langue ? Ce pronom pourrait tout autant correspondre aux lettrés et chercheurs chinois, voire à l'ensemble de la population de ce pays. Par conséquent, cette ambiguïté référentielle a pour effet de fondre le chercheur dans son groupe national. Le dernier pronom de l'extrait renforce encore cette impression : "nous les appelons » s'oppose à « ils ont leur (...) langages ». C'est ici le groupe ethnique majoritaire qui semble se poser comme co-locuteur, et le sujet scientifique s'estompe encore davantage. Li ne parviendrait pas à se singulariser en tant que chercheur distancié de son objet. Ce point est abordé incidemment par l'étudiant pendant l'entretien :

LI : (...) quand j'écris / je me mets toujours à la place d'un chercheur en fait

SF : mais tu es un chercheur

LI : sachant// oui sachant/ enfin /que oui/ donc un chercheur et puis donc / mais// QUAND MÊME dans ma thèse/ si tu veux parler du nous/ j'ai quand même différencié nous et je (...) parce que nous / si j'ai bien compris/ que nous on écrit HUMblement/ on voulait pas trop/voir/ (...) enfin on écrit de la façon humblement (...) même si t'as découvert une grande théorie (...)

L'embarras se devine dans la longue période d'hésitations et de faux départs du doctorant (sachant// oui sachant/ enfin /que oui/donc). Sa légitimité à se présenter en tant que chercheur est encore à construire (je me mets toujours à la place). "Écrire humblement », minimiser son apport personnel, quelle que soit la valeur du travail, apparaît comme une valeur fondamentale à ses yeux. Comment interpréter ce «nous on écrit »? Ne pourrait-il pas renvoyer à un groupe issu d'une même culture éducative, à l'origine de cette posture scientifique ? En tout état de cause, la confrontation entre cette tendance à l'effacement du sujet scientifique et les attentes des directeurs de thèse du laboratoire vis-à-vis des doctorants est éclairante : «Il faut assumer sa posture de chercheur » (M. Arnaud, corpus 3), « Il faut une certaine audace (...). Il y a une question de représentation de soi » (Mme Charles). La singularité, l'originalité, l'audace sont mises en avant. Ces attendus ne peuvent qu'entrer en tension avec le positionnement de Li, et ce qui apparaît comme des maladresses dans l'écriture révèle en réalité des conflits plus profonds.

Un dernier exemple fera apparaître une difficulté en quelque sorte inverse. Au moment d'évoquer la situation récente de son pays (partie contextuelle), Rahma (corpus 1) écrit :

Malgré les effets pernicieux, nocifs, vicieux et funestes de l'occupation, essayant de diviser définitivement le pays en trois, il existe une ouverture que l'Irak n'avait pas connue auparavant.

Abder, quant à lui, affirme : 
Sous prétexte de la sécurité nationale, Moubarak a dirigé le pays à travers le blocage politique et l'état d'urgence en vigueur sans interruption depuis son investiture le 14 octobre 1981. Il nous semble que ce président issu de l'armée n'a pas fait d'efforts, malgré ses promesses fréquentes, pour lancer une transition démocratique paisible dans le pays le plus peuplé du monde arabe.

Il est clair que ce qui semble depuis Weber, (1917) une règle d'airain de l'écriture scientifique, la neutralité axiologique, n'est pas observée ici. Le chercheur est censé se distancier de son objet et exprimer ses réserves par un emploi subtil de la modalisation, et l'excès de subjectivité est perçu comme une maladresse. L'entretien aborde ce point avec Rahma :

SF : quand vous dites ça, est-ce que c'est le chercheur qui parle ou bien le citoyen irakien (...)

RAHMA : les deux // parce que oui bien sûr c'est moi qui parle/ (...) JE suis Irakien/J'AI vécu là/ (...) et oui/ il y a l'effet de l'écriture personnelle bien sûr/ (...) c'est impossible de séparer le chercheur et le citoyen/ c'est une / capture imaginaire dans la tête

Pour lui, les choses sont claires : la distinction entre le chercheur spécialiste de l'Irak et le citoyen irakien est une fiction, un artefact, ce qu'il semble vouloir exprimer par l'expression « une capture imaginaire dans la tête ». C'est ce qu'il appelle "écriture personnelle » de la thèse, qui est, dans son cas, une thèse de réparation destinée à reconstruire son pays. Ici, la motivation elle-même de la thèse vient contredire le principe du discours objectivé. S'agit-il pour autant d'un attendu impératif de la part des enseignants-chercheurs ? Pour M. Arnaud (corpus 3), le doctorant doit « dire quelque chose, prendre parti, sans prendre position sur des sujets étrangers à la thèse. » Plus catégorique, M. Daniel affirme : "La neutralité axiologique dans l'écriture de la thèse est une illusion académique hypocrite. » M. Bernard, quant à lui, rappelle que dans la thèse «Il y a un sujet [écrivant], et donc une subjectivité qui peut et doit être contrôlée. » On note donc avec intérêt qu'aucun ne défend une neutralité axiologique stricto sensu, mais que globalement, une position d'équilibriste entre affirmation d'un point de vue et contrôle de la subjectivité est recommandée. Comprendre la subtilité d'un positionnement scientifique éloigné des postures scientifiques initiales du doctorant, voire en contradiction avec celles-ci, n'est donc pour lui pas le moindre des obstacles.

\section{Conclusion}

La mobilité académique au niveau doctoral présente donc des particularités, que ce soit au niveau des enjeux représentés par ce diplôme, de ses conséquences dans les parcours de vie, et des obstacles et des tensions que doivent résoudre les étudiants au quotidien. Ces premières analyses mettent en lumière l'intérêt d'une approche pluridimensionnelle de la didactique de l'écrit universitaire pour un public en situation de mobilité académique. La prise en compte des écarts entre les matrices rhétoriques et organisationnelles, ainsi que les postures scientifiques transmises et élaborées dans le système éducatif d'origine, d'une part, et celles attendues dans le système universitaire d'accueil, de l'autre, semble indispensable à la conception de formations aptes à satisfaire les différents besoins des étudiants internationaux. Le défi est cependant de taille, dans la mesure où le didacticien doit dégager et expliciter des normes et des 
usages de l'écrit en circulation dans chaque système, mais invisibles et inconnus car impensés par les sujets eux-mêmes. Comprendre les cultures académiques, y compris dans leur dimension cachée, revêt ainsi un caractère d'urgence, tant elles ont des retombées sur le parcours de recherche des doctorants internationaux et leur expérience de mobilité, mettant en jeu non seulement la réussite de leur doctorat mais aussi celle de leur projet de vie.

\section{Références bibliographiques}

Adam, J.-M. (2018). Le paragraphe: entre phrases et texte. Paris: A.Colin.

Allouche, V., \& Maurer, B. (2010). L'écrit en FLE: travail du style et maîtrise de la langue. Montpellier: Presses universitaires de la Méditerranée.

Anquetil, M., \& Molinié, M. (2008). L'expérience Erasmus au miroir de la réflexivité: penser et construire les acteurs sociaux. In G. Zarate, D. Lévy, \& C. Kramsch, Précis $d u$ plurilingusme et du pluriculturalisme. Paris : Archives contemporaines

Bardin, L. (1977). L'analyse de contenu (2e édition). Paris: Presses universitaires de France.

Barré-de Miniac, C., Brissaud, C., \& Rispail, M. (Éd.). (2005). La littéracie: conceptions théoriques et pratiques d'enseignement de la lecture-écriture. Paris: INRP

Bouchard, R. (2010). Les étudiants étrangers face à une production écrite « extra-ordinaire» : une thèse en français. Le français dans le monde-Recherches et applications, 123-132.

Bourdieu, P. (1979). Les trois états du capital culturel. Actes de la Recherche en Sciences Sociales, 30(1), 3-6. https://doi.org/10.3406/arss.1979.2654

Bourdieu, P. (1990). Les conditions sociales de la circulation internationale des idées. Romanistische Zeitschrift für Literaturgeschichtel Cahiers d'Histoire des Littératures Romanes, 14ème année, (1-2), 1-10.

Bourdieu, P., \& Passeron, J.-C. (1970). La reproduction: éléments pour une théorie du système d'enseignement. Paris: Minuit.

Cassin, B. (Éd.). (2004). Vocabulaire européen des philosophies: dictionnaire des intraduisibles. Paris: Le Robert : Seuil.

Coulon, A. (1997). Le métier d'étudiant: l'entrée dans la vie universitaire (Vol. 1-1). Paris: Economica.

Cuko, K. (2016). Perceptions de parents nouvellement arrivés dans le système scolaire québécois : la classe d'accueil, lieu de «révélation » de mobilités et d'immobilités dans le parcours d'insertion sociale. Cahiers internationaux de sociolinguistique, 2016/1(9), 263-290. 
Espagne, M. (2013). La notion de transfert culturel. Revue Sciences/Lettres, (1). https://doi.org/10.4000/rsl.219

Fløttum, K., \& Vold, E. (2010). L'éthos auto-attribué d'auteurs-doctorants dans le discours scientifique. LIDIL, 41, 41-58.

Gohard-Radenkovic, A. (1995). L'écrit: stratégies et pratiques. Paris: Clé international.

Gohard-Radenkovic, A. (1999). Communiquer en langue étrangère: de compétences culturelles vers des compétences linguistiques Bern : Peter Lang.

Gohard-Radenkovic, A. (2002). La culture universitaire comme culture en soi? Travaux neuchâtelois de linguistique, (36), 9-24.

Gohard-Radenkovic, A., \& Murphy-Lejeune, E. (2008). Mobilités et parcours. In G. Zarate, D. Lévy, \& C. Kramsch (Éd.), Précis du plurilinguisme et du multiculturalisme (p. 126-170).Paris: Archives contemporaines.

Goody, J. (1978). La raison graphique: la domestication de la pensée sauvage. (J. T. Bazin \& A. T. Bensa, Trad.) Paris : Minuit.

Hall, E. T. (1979). Au-delà de la culture. (M.-H. Hatchuel \& F. Graëve, Trad.). Paris: Seuil

INSEE. (2017). Tableaux de l'économie française (No. TEF 2017 102) (p. 100-101). Paris.

Kaufmann, J.-C. (2007). L'entretien compréhensif. (F. de Singly, Éd.). Paris: A. Colin.

Keller-Gerber, A. (2015). «Ces étudiants étrangers qui restent ou qui veulent rester»: résonance de discours en circulation sur l'immigration dans les récits d'étrangers diplômés en Suisse, candidats à «l'établissement » (Thèse de doctorat). Université de Fribourg (Suisse), Université de Franche-Comté (France), Fribourg, Besançon.

Latour, B., \& Woolgar, S. (1988). La vie de laboratoire: la production des faits scientifiques. (M. Biezunski, Trad.). Paris: La Découverte.

Lévy-Leblond, J.-M. (2006, mai 1). La science est-elle universelle ? Le Monde diplomatique $n^{\circ}$ 626, p. 32.

Licoppe, C. (1996). La formation de la pratique scientifique: le discours de l'expérience en France et en Angleterre, 1630-1820. Paris: La Découverte.

Madini, M. (2010). Quelques «lieux de rencontre» de Jean Peytard. Semen [en ligne], (29). Consulté à l'adresse http://journals.openedition.org/semen/8862

Murphy-Lejeune, E. (2003). L'étudiant européen voyageur: un nouvel étranger. Paris : Didier.

Papatsiba, V. (2003). Des étudiants européens: «Erasmus » et l'aventure de l'altérité. Berne : P.Lang. 
Pollet, M.-C. (2012). De la maîtrise du français aux littéracies dans l'enseignement supérieur (Vol. 1-1). Namur: Presses universitaires de Namur.

Pollet, M.-C. (2014). L'écrit scientifique à l'aune des littéracies universitaires: approches théoriques et pratiques. Namur: Presses universitaires de Namur.

Reuter, Y. (1998). De quelques obstacles à l'écriture de recherche. LIDIL, (17), 11-23.

Reuter, Y. (2004). Analyser les problèmes de l'écriture de recherche en formation. Pratiques, (121-122), 9-27.

Reuter, Y. (2012). Les didactiques et la question des littéracies universitaires. Pratiques, 153-154.

Weber, M. (1917). Le savant et le politique. (J. Freund, Trad.). Paris: Union générale d'éditions.

Weisser, M. (2010). Matrice disciplinaire et représentation de l'autre (p. 177-188), Représentations du transfrontalier, Presses universitaires de Nancy.

Zarate, G. (2005). L'expérience de la pluralité en situation de mobilité internationale dans la formation d'un capital plurilingue et pluriculturel. In O. Bertrand (Éd.), Diversités culturelles et apprentissage du français. Approche interculturelle et problématiques linguistiques Palaiseau.: Ecole Polytechnique. 